\title{
Consolidation therapy of arsenic trioxide alternated with chemotherapy achieves remarkable efficacy in newly diagnosed acute promyelocytic leukemia
}

\author{
This article was published in the following Dove Press journal: \\ OncoTargets and Therapy \\ 12 November 2015 \\ Number of times this article has been viewed
}

\author{
Cheng-cheng Liu' ${ }^{1-3, *}$ \\ Hua Wang ${ }^{1-3, *}$ \\ Wei-da Wangl-3 \\ Meng-yuan Zhul-3 \\ Qi-rong Geng ${ }^{1-3}$ \\ Yue Lu ${ }^{1-3}$ \\ 'Department of Hematological \\ Oncology, Sun Yat-sen University \\ Cancer Center, Guangzhou, People's \\ Republic of China; ${ }^{2}$ State Key \\ Laboratory of Oncology in South \\ China, Guangzhou, People's Republic \\ of China; ${ }^{3}$ Collaborative Innovation \\ Center for Cancer Medicine, \\ Guangzhou, People's Republic of China \\ *These authors contributed equally \\ to this work
}

\begin{abstract}
Background: Currently, all-trans retinoic acid (ATRA) combined with daunorubicin and ATRA combined with arsenic trioxide (ATO) therapies are considered the standard induction therapy regimens for adult patients newly diagnosed with acute promyelocytic leukemia (APL). However, there is no consensus concerning the optimal consolidation and maintenance therapies after induction therapy. In this study, we explored a new therapeutic strategy for APL that may be simple, effective, and safe.
\end{abstract}

Methods: The patients in our study were divided into high white blood cell (WBC) group and low WBC group according to the numeration of leukocytes at the first visit. The low WBC group received ATRA and ATO until complete remission (CR), and the high WBC group received anthracycline, ATRA, and ATO until CR. After achieving hematologic CR, ATO was alternated with chemotherapy for consolidation therapy. Three cycles were completed in the 1 st year with no maintenance therapy. The patients were followed for a median of 5 years after their initial treatment.

Results: After induction therapy, the rate of CR for the 18 patients was $100 \%$. The rate of negativity for the $P M L / R A R \alpha$ fusion gene following induction therapy was $100 \%$. There was no mortality during the treatment. Both the 5-year event-free survival rate and 5-year overall survival rate were $100 \%$. No relapses occurred during the follow-up period.

Conclusion: This study proposes a novel treatment for APL that is efficient, well-tolerated, and very simple to perform.

Keywords: acute promyelocytic leukemia, all-trans retinoic acid, arsenic trioxide, consolidation therapy, new therapeutic strategy, survival

\section{Introduction}

Acute promyelocytic leukemia (APL) is a distinct subtype of acute myeloid leukemia (AML) that is characterized by its morphology, $t(15 ; 17)$ translocation leading to the formation of the PML/RAR $\alpha$ fusion gene, and life-threatening coagulopathy. ${ }^{1}$ The prognosis of APL has changed from the worst among AMLs to currently the best among AMLs due to the application of all-trans retinoic acid (ATRA) and arsenic trioxide (ATO). ${ }^{2-5}$ A consensus has been reached that ATRA alone or in combination with ATO, with the proper addition of anthracyclines according to white blood cell (WBC) count, is able to achieve complete remission (CR) and results in negativity for the PML/RAR $\alpha$ fusion gene. ${ }^{6,7}$ Currently, there is no consensus regarding the optimal consolidation and maintenance therapies after induction therapy. Additionally, whether the early involvement of maintenance therapy benefits low-risk patients who
Correspondence: Yue Lu

Department of Hematological Oncology, Sun Yat-sen University Cancer Center; State Key Laboratory of Oncology in South China; Collaborative Innovation Center for Cancer Medicine, 65I Dongfeng East Road, 510060 Guangzhou, Guangdong, People's Republic of China Tel +86 2087343145

Fax +86 2087343145

Email lvyue@sysucc.org.cn 
have already achieved molecular CR is still being debated. Because the regimen administered after remission determines the long-term survival rate of APL, in this article, we explored an effective, safe, and simple therapeutic strategy for APL that would potentially provide a remedy to this issue.

\section{Patients and methods}

\section{Ethics statement}

This study was approved by the Institutional Review Board of Sun Yat-sen University Cancer Center, and written informed consent was obtained from every volunteer and patient prior to treatment. Additionally, this study was conducted in accordance with the Declaration of Helsinki.

\section{Patients}

The patients included in this study met the following criteria: 1) newly diagnosed APL in accordance with the morphological criteria (M0-M7) of the French-AmericanBritish classification system for myelocytic leukemias; ${ }^{8}$ 2) confirmation of APL diagnosis by both a cytogenetic assay for $\mathrm{t}(15 ; 17)(\mathrm{q} 24 ; \mathrm{q} 21)$ and a reverse transcription polymerase chain reaction assay for PML/RAR $\alpha$; 3) the patients had completed the whole induction therapy and consolidation therapy. In our country, due to the economic and other reasons, some patients could not complete the treatment, or before the completion of the treatment, serious cerebral hemorrhage and serious infections occurred that led to the early death of the patients. The patients with APL who were transferred to our hospital on relapse were not included in our study. The patients included in our study were the ones who achieved the complete treatment.

Eighteen patients initially diagnosed with adult APL were enrolled from January 2002 to December 2012 at the Sun Yat-sen University Cancer Center. The patients received regular treatment. To evaluate the efficacy and safety of the treatment, complete data of 18 cases (including ten males and eight females, median age: 34 years; range: 19-64 years; refer Table 1 for the patients' clinical details) were analyzed and summarized in this long-term and systematic follow-up study. The patients were followed for a median of 5 years after their initial treatment. The deadline for follow-up was December 2014.

\section{Treatment strategies}

According to the classification system of Sanz et al, ${ }^{9}$ patients can be divided into three groups based on their WBC count: low risk of relapse $\left(\mathrm{WBC}\right.$ count $<10 \times 10^{9} / \mathrm{L}$, platelet count $\left.>40 \times 10^{9} / \mathrm{L}\right)$, intermediate risk of relapse (WBC count $<10 \times 10^{9} / \mathrm{L}$, platelet count $40 \times 10^{9} / \mathrm{L}$ ), and high risk of relapse (WBC count $\geq 10 \times 10^{9} / \mathrm{L}$, platelet count $\leq 40 \times 10^{9} /$ L) (Table 1$)$.
Table I Clinical characteristics at baseline

\begin{tabular}{|c|c|c|}
\hline Characteristic & $\mathbf{n}$ & $\%$ \\
\hline \multicolumn{3}{|l|}{ Age, years } \\
\hline Median (range) & $34(19-65)$ & \\
\hline$<40$ & II & 61.1 \\
\hline$\geq 40$ & 7 & 38.9 \\
\hline \multicolumn{3}{|l|}{ Sex } \\
\hline Male & 10 & 55.6 \\
\hline Female & 8 & 44.4 \\
\hline \multicolumn{3}{|l|}{ WBC count $\left(\times 10^{9}\right)$} \\
\hline Median (range) & $2.02(0.3-77.8)$ & \\
\hline$<10$ & 13 & 72.2 \\
\hline$\geq 10$ & 5 & 27.8 \\
\hline \multicolumn{3}{|l|}{ PLT count $\left(\times 10^{9}\right)$} \\
\hline Median (range) & $33(11-133)$ & \\
\hline$\leq 40$ & 12 & 66.7 \\
\hline$>40$ & 6 & 33.3 \\
\hline \multicolumn{3}{|l|}{$\mathrm{LDH}(\mathrm{U} / \mathrm{L})$} \\
\hline Median (range) & $196(96.8-816)$ & \\
\hline Normal & 13 & 72.2 \\
\hline$>$ normal & 5 & 27.8 \\
\hline \multicolumn{3}{|l|}{ Risk stratification } \\
\hline Low risk & 6 & 33.3 \\
\hline Intermediate risk & 7 & 38.9 \\
\hline High risk & 5 & 27.8 \\
\hline Hemoglobin ( $g / L)$, median (range) & $74(47-115)$ & \\
\hline $\operatorname{RBC}\left(\times 10^{9}\right)$, median (range) & $2.5(1.2-3.8)$ & \\
\hline Peripheral promyelocytes (\%), median (range) & $67(25-92)$ & \\
\hline Marrow promyelocytes (\%), median (range) & $77.5(28.8-91.5)$ & \\
\hline Fibrinogen $(\mathrm{g} / \mathrm{L})$, median (range) & $1.6(0.4-5.8)$ & \\
\hline
\end{tabular}

Abbreviations: $\mathrm{LDH}$, lactic dehydrogenase; PLT, platelet; RBC, red blood cell; WBC, white blood cell.

Our induction therapy was administered as follows: $30 \mathrm{mg} / \mathrm{m}^{2}$ ATRA orally per day and $0.16 \mathrm{mg} / \mathrm{kg}$ ATO intravenously per day until CR. When the patient's WBC count exceeded $10 \times 10^{9} / \mathrm{L}$, the patient was given hydroxyurea (daily doses of 20-40 mg/kg) or daunorubicin and cytarabine (Ara-c)/idarubicin and cytarabine regimen (daunorubicin: $60 \mathrm{mg} / \mathrm{m}^{2}$ per day for 3 days and Ara-C: $100 \mathrm{mg} / \mathrm{m}^{2}$ per day for 3-5 days, or idarubicin: $10 \mathrm{mg} / \mathrm{m}^{2}$ per day for 3 days and Ara-C: $100 \mathrm{mg} / \mathrm{m}^{2}$ per day for 3-5 days) until the patient's WBC count $<10 \times 10^{9} / \mathrm{L}$ (Figure 1 ).

Our consolidation therapy was administered as follows: ATO alternated with chemotherapy during consolidation therapy after hematologic CR. Three cycles were completed in the 1st year. Each cycle included the daunorubicin plus ATRA regimen (daunorubicin, $60 \mathrm{mg} / \mathrm{m}^{2}$ per day [days 1-3] and ATRA $30 \mathrm{mg} / \mathrm{m}^{2}$ per day [days 1-28]) or the idarubicin plus ATRA regimen (idarubicin $10 \mathrm{mg} / \mathrm{m}^{2}$ per day [days 1-3] and ATRA $30 \mathrm{mg} / \mathrm{m}^{2}$ per day [days 1-28]), plus the ATO regimen $(0.16 \mathrm{mg} / \mathrm{kg}$ per day for 14 days). In addition, high-risk patients received one more course of high-dose Ara-C $\left(3 \mathrm{~g} / \mathrm{m}^{2}\right)$ at the end of consolidation therapy (Figure 1).

To avoid central nervous system relapse, all patients received at least once intrathecal injection of three drugs 


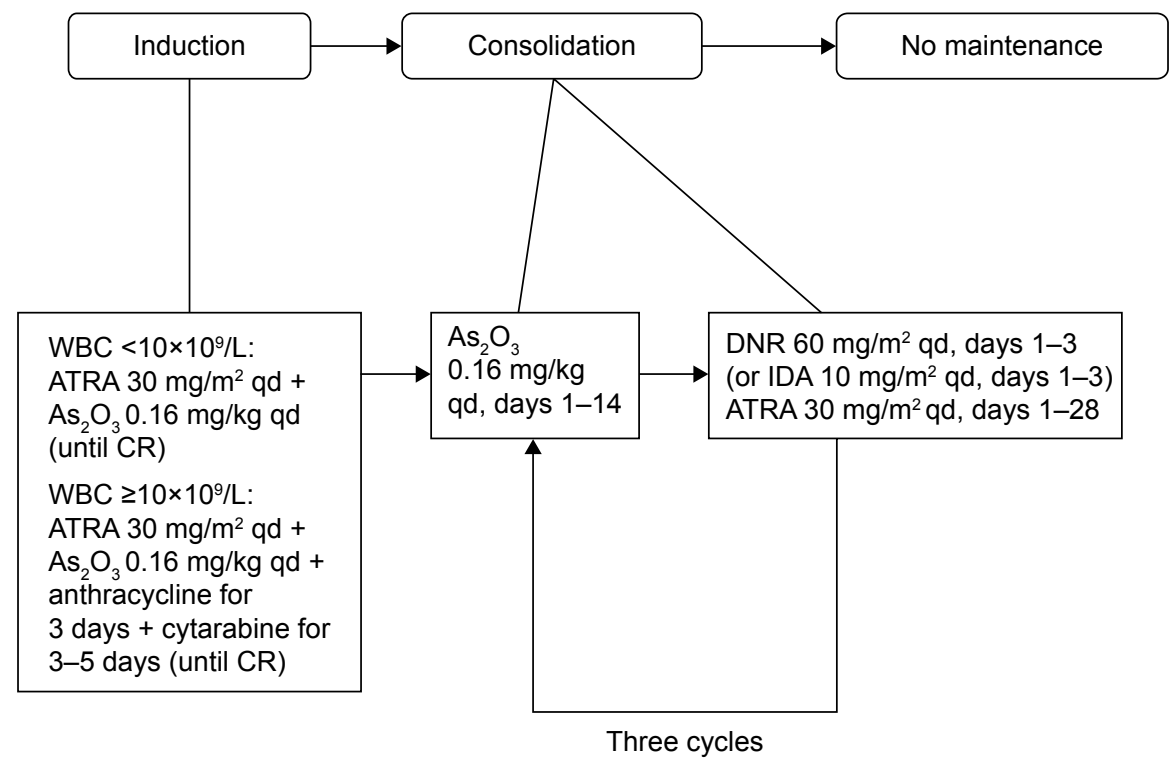

Figure I Flowchart depicting the detailed information of this regimen.

Abbreviations: ATRA, all-trans retinoic acid; CR, complete remission; DNR, daunorubicin; IDA, idarubicin; qd, every day; WBC, white blood cell.

containing methotrexate (10-15 mg), dexamethasone (5 mg) or prednisolone (40 mg), and Ara-C (40-50 mg) during their consolidation therapy. Coagulation and fibrinolysis parameters, such as fibrinogen, fibrin degradation product, D-dimer level, prothrombin time, and activated partial thromboplastin time, were monitored to direct the use of low-dose heparin, platelet transfusion, and fresh plasma when necessary. Hepatic toxicity was given particular caution as follows: ATO should be decreased to half the original dose $(0.08 \mathrm{mg} / \mathrm{kg}$ per day) in the case of grade $0-1$ liver dysfunction and ought to be withdrawn immediately in the case of grade $2-4$ liver dysfunction. The patients with a suspected APL differentiation syndrome received dexamethasone treatment.

There was no maintenance therapy. After induction and consolidation therapy, bone marrow samples were obtained every 3 months for the first 2 years and every 6 months from 2 to 5 years after CR. The PML/RAR $\alpha$ fusion gene was regularly monitored every 6 months for 3 years after the consolidation therapy was completed.

\section{Definitions}

The main end points analyzed were CR, event-free survival (EFS), and overall survival (OS). Hematologic CR, molecular $\mathrm{CR}$, hematologic relapse, and molecular relapse were defined according to the criteria recommended by the US National Cancer Institute. EFS for CR patients was the time to the observation of an event, for example, failure to achieve $\mathrm{CR}$, relapse after achieving $\mathrm{CR}$, such as hematologic relapse, molecular relapse, extramedullary relapse, and death. The OS was defined as the time interval from the date of the initial diagnosis to death from any cause or to the last follow-up in censored patients.

\section{Results \\ Patient characteristics}

In total, 18 patients (ten males and eight females) with a median age of 34 years were included in this study. The median follow-up time was 52 months (range: 24-122 months). The laboratory test results of the 18 patients revealed that the median WBC count was $2.02 \times 10^{9} / \mathrm{L}$ (range: $0.3 \times 10^{9} / \mathrm{L}$ to $77.8 \times 10^{9} / \mathrm{L}$ ), the median hemoglobin level was $74 \mathrm{~g} / \mathrm{L}$ (range: $47-115 \mathrm{~g} / \mathrm{L}$ ), and the median platelet count was $33 \times 10^{9} / \mathrm{L}$ (range: $11 \times 10^{9} / \mathrm{L}$ to $133 \times 10^{9} / \mathrm{L}$ ). Six patients were at low risk, seven were at intermediate risk, and five were at high risk. Elevated lactic dehydrogenase level $(>245 \mathrm{U} / \mathrm{L})$ was observed in $27.8 \%$ of the patients. The median peripheral promyelocyte count was $67 \%$ (range: $25 \%-92 \%$ ), and the median marrow promyelocyte count was $77.5 \%$ (range: $28.8 \%-91.5 \%$ ) (Table 1 ).

\section{Treatment outcome}

All 18 patients finished the induction regimen and were evaluated for response. None of the patients died as a result of the treatment. After induction therapy, the CR rate of the 18 patients was $100 \%$. The rate of PML/RAR $\alpha$ fusion gene negativity was $100 \%$. The estimated median duration of EFS and OS could not be determined, as the 5-year EFS rate and the 5-year OS rate were both $100 \%$. No relapse occurred during the follow-up period (Table 2, Figures 2 and 3). 
Table 2 Induction response

\begin{tabular}{ll}
\hline Clinical parameter & All patients (N=18) \\
\hline Hematologic CR, number (\%) & $18(100.0)$ \\
Molecular CR, number (\%) & $18(100.0)$ \\
$\begin{array}{l}\text { Time from treatment to hematologic CR (days), } \\
\text { median (range) }\end{array}$ & $28(17-48)$ \\
$\begin{array}{l}\text { Time from treatment to molecular CR (days), } \\
\text { median (range) }\end{array}$ & $48(38-70)$ \\
$\begin{array}{l}\text { Differentiation syndrome, number (\%) } \\
\text { Duration of hyperleukocytosis (days), } \\
\text { median (range) }\end{array}$ & $\mathrm{I}(5.6)$ \\
\hline
\end{tabular}

Abbreviation: $\mathrm{CR}$, complete remission.

\section{Toxicity}

There was no mortality during treatment. Gastrointestinal adverse reactions occurred in eight patients. No serious cardiotoxicity was observed. Differentiation syndrome occurred in one patient. Liver function tests showed increased levels of alanine transaminase and aspartate transaminase in five cases, but these levels reduced to the normal range after the treatment for 1-2 weeks with liver-protecting drugs. No treatment interruption or dose reduction occurred due to liver toxicity. Fever occurred in $55.6 \%$ of the patients, and bone pain was experienced by $16.7 \%$ of the patients. Skin reaction was observed in $5.6 \%$ of the patients. None of the patients developed a secondary neoplasm during the follow-up period (Table 3 ).

\section{Discussion}

Currently, APL is a subtype of AML that does not require stem cell transplantation for treatment, as conventional chemotherapy alone is sufficient to cure APL. The advent of ATRA and ATO for the treatment of APL allowed the rate of CR to reach $90 \%$, but it is also associated with a relapse rate of $15 \%-25 \% .^{10-13}$ The physiopathology of APL is a maturation arrest in the promyelocytic stage caused by the PML-RAR $\alpha$

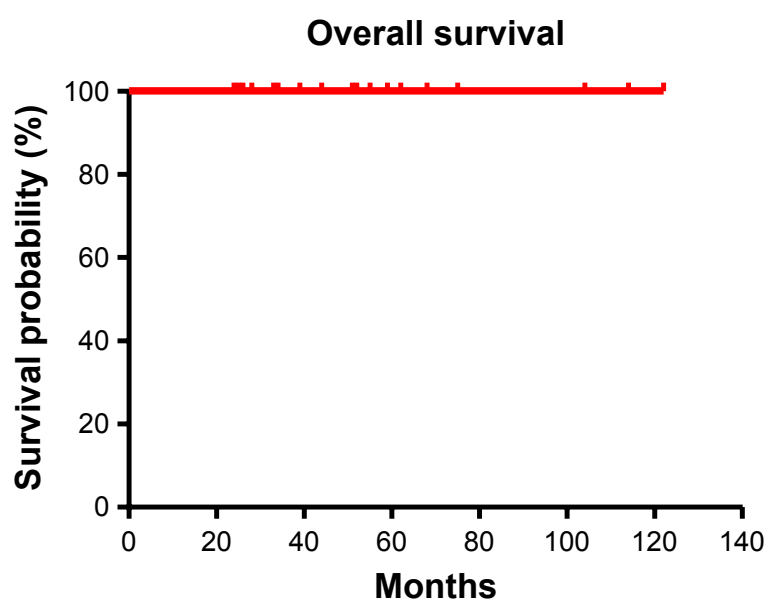

Figure 2 Kaplan-Meier curve of overall survival.

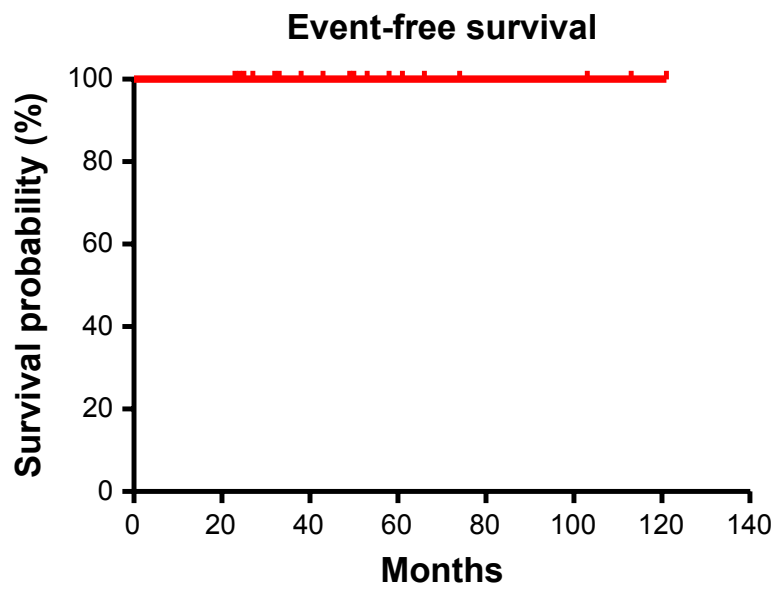

Figure 3 Kaplan-Meier curve of event-free survival.

chimeric protein. ${ }^{14,15}$ First discovered in 1980, ATRA induces differentiation of leukemic promyelocytes into mature granulocytes by ligation of the PML-RAR $\alpha$ receptor. ${ }^{16}$ Moreover, ATO binds to the PML-RAR $\alpha$ protein and causes the dosedependent degradation or apoptosis of APL cells. During induction therapy with these two drugs (ATRA+ATO), the patients can achieve $\mathrm{CR}$, but the inability of these two drugs to remove minimal residual leukemia can lead to relapse. ${ }^{17-19}$ Studies ${ }^{20,21}$ have shown that mice achieved CR after treatment with ATRA and ATO appeared to reproduce the PML-RAR $\alpha$ fusion gene after receiving bortezomib (a proteasome inhibitor) treatment, which suggests that leukemic stem cells are not cleared by ATRA and ATO in mice. Further study showed that ATO should be combined with standard chemotherapy to clear minimal residual leukemia. ${ }^{22}$

A meta-analysis of adults with APL demonstrated that the use of ATRA and ATO compared to ATO alone increased the rate of $C R$, shortened the time to remission, and generated a better remission rate during 1 year of follow-up. ${ }^{23}$ In a study published in 2013, Lo-Coco et al $^{13}$ observed that ATRA and ATO were not inferior to ATRA with chemotherapy in cases of newly diagnosed adults with APL. The combination of ATRA and ATO is currently approved in North America and Europe for the treatment of newly diagnosed and relapsed adults with APL. The consensus has been reached that ATRA

Table 3 Toxicity profile

\begin{tabular}{lll}
\hline Toxicity & $\mathbf{n}$ & $\%$ \\
\hline Liver dysfunction & 5 & 27.8 \\
Cardiac arrhythmia & 2 & II.I \\
Gastrointestinal adverse reaction & 8 & 44.4 \\
Bone pain & 3 & 16.7 \\
Skin reaction & $\mathrm{I}$ & 5.6 \\
Fever & 10 & 55.6 \\
\hline
\end{tabular}


combined with daunorubicin and ATRA combined with ATO are standard induction therapy regimens for adult patients newly diagnosed with APL. Therefore, in our study, the low WBC group received ATRA and ATO, and the high WBC group received anthracycline, ATRA, and ATO. It turned out that we achieved a high rate of CR.

At present, the main focus of APL treatment is on the consolidation and maintenance therapy. The primary goal of medical staff is to reduce the costs of treatment and the pain associated with it, while maintaining a high long-term EFS rate and improving patient prognosis. Xin et $\mathrm{al}^{6}$ suggested that the regimen administered after remission determines the long-term survival rate. The long-term survival rate was lower in patients treated with chemotherapy alone. Chemotherapy combined with ATRA or ATO showed a significantly improved OS rate and recurrence-free survival rate compared with chemotherapy alone. The highest OS rate was achieved when ATRA, ATO, and chemotherapy are combined and used alternatively. Due to the limitations of ATRA and ATO, the use of chemotherapeutic drugs in the consolidation therapy regimen cannot be ignored. ${ }^{24,25}$

Our study is unique in that it incorporates consolidation therapy instead of maintenance therapy: ATO and chemotherapy are used in turn during consolidation therapy after hematologic CR. As we know, the main limitation of anthracycline is the risk of cardiotoxicity with cumulative doses. For children and elderly patients who are physically weak, bone marrow suppression and secondary severe infections are likely to occur after stronger standard chemotherapy, and the treatment-related mortality with this therapy is high. In our study, we used ATO alternated with conventional chemotherapy. With this strategy, the bone marrow of patients has a relatively long time to recover, which can help patients, particularly those who are weak, to better tolerate their next course of treatment. ATO has been proven to be quite safe. When compared with anthracyclines, bone marrow myelosuppression by ATO appears to be minor. The common adverse effects of ATO are grade 1-2 hepatotoxicity, gastrointestinal adverse reactions, neurotoxicity, and very rarely, grade 3-4 hepatotoxicity, and these adverse effects are usually reversible. ${ }^{15,26,27}$ In our study, the treatment was not discontinued in any patients due to ATO toxicity. ATO was alternated with chemotherapy, which allowed the interval between ATO treatments to be extended, and avoided the deposition of ATO. In our study, ATO was found to be quite safe.

This treatment program takes a total of 7 months, including 1 month of induction therapy and 6 months of ATO combined with sequential chemotherapy. The patients were regularly monitored for the presence or absence of PML-RAR $\alpha$ fusion gene every 6 months for 2 years after consolidation therapy was completed with no further maintenance therapy. The molecular biology of APL cells changes 3 months before hematologic relapse or extramedullary relapse. Therefore, after consolidation therapy, the patients should be regularly monitored for the presence or absence of PML-RAR $\alpha$ fusion gene. Once the PML-RAR $\alpha$ fusion gene is observed, which suggests the risk of early recurrence, intervention must be implemented as soon as possible. For patients and doctors, this treatment program is simple, well planned, and achieved good patient compliance. Moreover, in our study, the rate of CR for the 18 patients was $100 \%$, and the rate of PML/RAR $\alpha$ fusion gene negativity was $100 \%$. Both the 5 -year EFS rate and the 5 -year OS rate were $100 \%$. There was no mortality and no relapse during the follow-up period. Although chemofree induction and consolidation therapy using ATRA/ATO in Lo Cocco's NEJM paper acquire surprising results, the follow-up time is not very long. ${ }^{13}$ This regimen was indeed proven to be a very effective and convenient therapy regimen with lower toxicity compared with most therapy regimens reported previously. ${ }^{12,28}$

In summary, the regimen in our study had many advantages. First, this regimen prolonged the interval between standard chemotherapy cycles, effectively reducing the incidence of severe bone marrow suppression after chemotherapy and lowering the incidence of serious infections. Second, this regimen extended the interval between ATO treatments, avoiding the deposition of ATO. Third, this regimen significantly reduced the patient's total medical expenses due to the shortened treatment duration.

Our aim is to deliver the most timely and best quality treatment to each newly diagnosed patients with APL. However, for low-risk patients, ATO+ATRA combined with less chemotherapy may be a more appropriate choice. For high-risk patients, we are still searching for a better treatment regimen: in a follow-up series of clinical trials, we will examine whether median-dose or high-dose chemotherapy combined with ATO is better for the treatment of high-risk patients.

Our results confirmed that this chemotherapy regimen achieved ideal outcomes in patients with APL. However, the time and cost of three consecutive ATO treatments and three courses of standard chemotherapy are still unbearable for some patients. It would be even more appealing if the treatment regimen could achieve the same efficacy with fewer ATO treatments and standard chemotherapy cycles, or the use of oral medications. Finding an approach to achieve this would be our goal in the further study. 


\section{Conclusion}

This study presented a simple treatment regimen for patients with APL with impressive efficacy and high tolerability. Compared with other treatments discussed in other reports in the literature, this regimen has obvious advantages (such as being simple, effective, and safe). The most notable advantages are the high EFS and OS rates and no relapse during the follow-up period. The efficacy was so great that this regimen may be considered a breakthrough in the treatment of APL. The disadvantage of our study is that the number of cases is small, so more studies are necessary to confirm our findings. The next steps are to expand the samples of cases, simplify the treatment plan, and design a more costeffective treatment.

\section{Acknowledgments}

The authors thank all the investigators involved in this study, including the physicians, nurses, pathologists, and laboratory technicians. This work was supported by the following funds: National Natural Science Foundation of China (contract/grant numbers 30471976 and 81272620) and Science and Technology projects of Guangdong Province (contract/grant numbers 2010B031600233 and 2010A090200019). The funders had no role in study design, data collection and analysis, decision to publish, or preparation of the manuscript.

\section{Disclosure}

The authors report no conflicts of interest in this work.

\section{References}

1. Hurd DD, Vukelich M, Arthur DC, et al. 15;17 Translocation in acute promyelocytic leukemia. Cancer Genet Cytogenet. 1982;6:331-337.

2. Castaigne S, Chomienne C, Daniel MT, et al. All-trans retinoic acid as a differentiation therapy for acute promyelocytic leukemia. I. Clinical results. Blood. 1990;76:1704-1709.

3. Warrell RJ, Frankel SR, Miller WJ, et al. Differentiation therapy of acute promyelocytic leukemia with tretinoin (all-trans-retinoic acid). $N$ Engl J Med. 1991;324:1385-1393.

4. Wang ZY, Chen Z. Acute promyelocytic leukemia: from highly fatal to highly curable. Blood. 2008;111:2505-2515.

5. Shen ZX, Shi ZZ, Fang J, et al. All-trans retinoic acid/As2O3 combination yields a high quality remission and survival in newly diagnosed acute promyelocytic leukemia. Proc Natl Acad Sci U S A. 2004;101:5328-5335.

6. Xin L, Wan-jun S, Zeng-jun L, et al. A survival study and prognostic factors analysis on acute promyelocytic leukemia at a single center. Leuk Res. 2007;31:765-771.

7. Ades L, Sanz MA, Chevret S, et al. Treatment of newly diagnosed acute promyelocytic leukemia (APL): a comparison of French-Belgian-Swiss and PETHEMA results. Blood. 2008;111:1078-1084.

8. Castoldi GL, Liso V, Specchia G, Tomasi P. Acute promyelocytic leukemia: morphological aspects. Leukemia. 1994;8 Suppl 2: S27-S32.
9. Sanz MA, Martin G, Diaz-Mediavilla J. All-trans-retinoic acid in acute promyelocytic leukemia. $N$ Engl J Med. 1998;338:393-394.

10. Ades L, Guerci A, Raffoux E, et al. Very long-term outcome of acute promyelocytic leukemia after treatment with all-trans retinoic acid and chemotherapy: the European APL Group experience. Blood. 2010;115: 1690-1696.

11. Quezada G, Kopp L, Estey E, Wells RJ. All-trans-retinoic acid and arsenic trioxide as initial therapy for acute promyelocytic leukemia. Pediatr Blood Cancer. 2008;51:133-135.

12. Lo-Coco F, Cicconi L. What is the standard regimen for patients with acute promyelocytic leukemia? Curr Hematol Malig Rep. 2014;9: 138-143.

13. Lo-Coco F, Avvisati G, Vignetti M, et al. Retinoic acid and arsenic trioxide for acute promyelocytic leukemia. N Engl J Med. 2013;369: $111-121$.

14. Diverio D, Pandolfi PP, Biondi A, et al. Absence of reverse transcription-polymerase chain reaction detectable residual disease in patients with acute promyelocytic leukemia in long-term remission. Blood. 1993;82:3556-3559.

15. Dos SG, Kats L, Pandolfi PP. Synergy against PML-RARa: targeting transcription, proteolysis, differentiation, and self-renewal in acute promyelocytic leukemia. J Exp Med. 2013;210:2793-2802.

16. Kakizuka A, Miller WJ, Umesono K, et al. Chromosomal translocation $\mathrm{t}(15 ; 17)$ in human acute promyelocytic leukemia fuses RAR alpha with a novel putative transcription factor, PML. Cell. 1991;66:663-674.

17. Tallman MS. The expanding role of arsenic in acute promyelocytic leukemia. Semin Hematol. 2008;45:S25-S29.

18. Bonati A, Rizzoli V, Lunghi P. Arsenic trioxide in hematological malignancies: the new discovery of an ancient drug. Curr Pharm Biotechnol. 2006;7:397-405.

19. Chen GQ, Shi XG, Tang W, et al. Use of arsenic trioxide (As2O3) in the treatment of acute promyelocytic leukemia (APL): I. As2O3 exerts dosedependent dual effects on APL cells. Blood. 1997;89:3345-3353.

20. Rego EM, Pandolfi PP. Analysis of the molecular genetics of acute promyelocytic leukemia in mouse models. Semin Hematol. 2001;38:54-70.

21. Lo CF, Ammatuna E, Noguera N. Treatment of acute promyelocytic leukemia with gemtuzumab ozogamicin. Clin Adv Hematol Oncol. 2006:4:57-62, 76-77.

22. Raffoux E, Rousselot P, Poupon J, et al. Combined treatment with arsenic trioxide and all-trans-retinoic acid in patients with relapsed acute promyelocytic leukemia. J Clin Oncol. 2003;21:2326-2334.

23. Xu SN, Chen JP, Liu JP, Xia Y. Arsenic trioxide in combination with alltrans retinoic acid for acute promyelocytic leukemia: a systematic review and meta-analysis. Zhong Xi Yi Jie He Xue Bao. 2009;7:1024-1034.

24. Hu J, Liu YF, Wu CF, et al. Long-term efficacy and safety of alltrans retinoic acid/arsenic trioxide-based therapy in newly diagnosed acute promyelocytic leukemia. Proc Natl Acad Sci U S A. 2009;106: 3342-3347.

25. Sanz MA, Montesinos P, Rayon C, et al. Risk-adapted treatment of acute promyelocytic leukemia based on all-trans retinoic acid and anthracycline with addition of cytarabine in consolidation therapy for high-risk patients: further improvements in treatment outcome. Blood. 2010;115:5137-5146

26. Zhou J, Zhang Y, Li J, et al. Single-agent arsenic trioxide in the treatment of children with newly diagnosed acute promyelocytic leukemia. Blood. 2010;115:1697-1702.

27. Coutre SE, Othus M, Powell B, et al. Arsenic trioxide during consolidation for patients with previously untreated low/intermediate risk acute promyelocytic leukaemia may eliminate the need for maintenance therapy. Br J Haematol. 2014;165:497-503.

28. Coombs CC, Tavakkoli M, Tallman MS. Acute promyelocytic leukemia: where did we start, where are we now, and the future. Blood Cancer J. 2015;5:e304. 
OncoTargets and Therapy

\section{Publish your work in this journal}

OncoTargets and Therapy is an international, peer-reviewed, open access journal focusing on the pathological basis of all cancers, potential targets for therapy and treatment protocols employed to improve the management of cancer patients. The journal also focuses on the impact of management programs and new therapeutic agents and protocols on The manuscript management system is completely online and includes a very quick and fair peer-review system, which is all easy to use. Visit http://www.dovepress.com/testimonials.php to read real quotes from published authors.

Submit your manuscript here: http://www.dovepress.com/oncotargets-and-therapy-journal 